\title{
NUEVAS PAUTAS DE MIGRACIÓN Y POBLAMIENTO: LAS DINÁMICAS DE LOS MUNICIPIOS DE CATALUÑA ${ }^{1, *}$
}

\author{
Solana Solana, M. \\ Departament de Geografia. Universitat Autònoma de Barcelona
}

\begin{abstract}
Resumen: Cataluña muestra cambios significativos en el sistema de poblamiento. La concentración de la población ha dado lugar a una dispersión creciente que tiene como principal destino los municipios de menos de 10.000 habitantes -habitualmente clasificados como intermedios o rurales-. Si bien en un primer momento dicha dispersión se concentraba en la Región Metropolitana de Barcelona, en los últimos años se asiste a una progresiva difusión de la población hacia áreas cada vez más alejadas del Barcelonés. Sin embargo, no es un proceso generalizado en todo el territorio catalán. Estos cambios son producto básicamente de la migración, actuando el Barcelonés y las principales ciudades de su entorno como origen. En esta comunicación se analizan estos procesos que suponen una transformación importante del sistema de poblamiento en el territorio. Un estudio más pormenorizado de los factores y de los impactos sociales y económicos que tiene esta migración está pendiente de realizarse. También se apuntan algunas líneas de reflexión que, a raíz de la literatura científica europea, pueden servir como elementos de guía para el análisis de estos procesos en el caso de Cataluña y España.
\end{abstract}

Palabras clave: migración, poblamiento, Cataluña, análisis municipal

\begin{abstract}
Cataluna exhibits important settlement changes. Population concentration has been replaced by a growing population dispersal, which main destination are municipalities above 10.000 inhabitants - usually classified as "intermedios" or "rurals"- At first, this population dispersal was concentrated in the Región Metropolitana de Barcelona, but during last years there is a growing population dispersal in more remote areas from Barcelonès. Nevertheless, it is not a common process in the whole Cataluña. These changes are basically produced by migration. Barcelonès a.nd the most populated cities which surround it are the main origin. This paper analyses these processes that are transforming the Catalan area. It is needed a more detailed study about causes and economical and social effects this migration is producing. It is also pointed out some lines of research derived from other European examples, but they can help us as reference points to develop the analysis of these processes in Catalonia and Spain.
\end{abstract}

Key words: migration, settlement, Catalonia, territorial analysis

* Recibido: 10-11-03. Aceptado: 10-12-04. 


\section{Migración y poblamiento: líneas de investigación sobre las dinámicas territoriales de las áreas rurales}

El cambio en las pautas de localización de la actividad económica y de la población a escala regional es un tema sobre cuya naturaleza surgen numerosos interrogantes. En el trasfondo nos encontramos básicamente con las nuevas funciones y actividades que adquieren los diferentes espacios, por ejemplo urbanos o metropolitanos frente a los no metropolitanos, su jerarquización e interrelaciones.

La ciudad y lo urbano han sido objeto prioritario en el estudio de las transformaciones territoriales que han tenido lugar durante el proceso de urbanización de la población y la adopción de un modo de vida urbano. En contraposición, el espacio rural se ha tendido a definir de manera negativa, como aquello que no es urbano, $y$ su entrada en el estudio de la transformación territorial ha venido de la mano de los intensos procesos de despoblación, subordinación y pérdida de dinamismo económico frente a la ciudad.

No es el objetivo de esta comunicación entrar a fondo en un análisis tan complicado como es la definición de conceptos ambiguos y cargados de múltiples significados como es "urbano" o "rural". La propia presentación que señala los objetivos de esta ponencia ya da cuenta de las dificultades que plantea un tratamiento dualístico del territorio. Los intentos por definir lo que es rural y la creación de tipologías sobre el espacio rural tienen una amplia tradición en otros países (Cloke, 1977; INRA-INSEE, 1990; Kayser, 1990, 1993). En el caso de España las aproximaciones teóricas hacia el concepto y su definición son más escasas (Estébanez, 1988, García Ramon, Tulla, Valdovinos, 1995). Ello hace conveniente tomar como punto de referencia los planteamientos elaborados desde otras realidades históricas, otras formas de organización del territorio y una dinámica diferente en la interacción de los diferentes espacios. A pesar de su interés, deben ser en todo caso amoldadas a las características propias de las sociedades del Sur de Europa. Esta importación de referencias y líneas de investigación también podría llevar al peligro de una excesiva desvinculación de la reflexión desde la geografía española sobre las dinámicas territoriales propias. Pero también es cierto que los grupos de investigación geográficos británicos y franceses, básicamente, han aportado elementos de interés al debate sobre los procesos de transformación territorial en las sociedades occidentales y, en concreto, sobre la nueva configuración, dinámica y características de aquello que se define como el campo, lo rural o el espacio rural.

Por una parte, la ya apuntada definición de lo que es rural se presenta de manera problemática. La contraposición dicotómica entre urbano y rural tiende a ocultar un grado de interrelación mucho más importante del que se acostumbra a utilizar e incluso la imposibilidad de establecer dos categorías diferenciadas (Hoggart, 1988, 
1990; Halfacree, 1991, 1993, 1994, 1995). No es ajeno a este planteamiento la aparición con fuerza en el ámbito de la geografía anglosajona del concepto del espacio rural post-productivista (AGE; Fielding, 1990, Halfacree, 1997). La pérdida de la agricultura como base económica y punto de referencia y definición del espacio rural dibuja un espacio nuevo en el cual la función prioritaria se circunscribe a servir de residencia a un porcentaje cada vez más importante de la población. En este sentido, desde la geografía española se viene estudiando este proceso de transformación económica y la aparición de nuevas actividades dinamizadoras del espacio rural, caso del turismo o algunas actividades relacionadas con la industria. La geografía que se realiza en nuestro país ha primado hasta ahora el factor económico en detrimento de los cambios sociales y culturales. Así, fenómenos como los procesos de cambio social en las âreas rurales (Cloke, Phillips, Thrift, 1995. Murdoch, 1995, Phillips, 1993, Urry, 1995), que han llevado a determinados autores a utilizar el término de gentrificación (Champion, Coombes, Fotheringham, 1998; Cloke, Thrift, 1990, Cloke, Phillips, Thrift, 1995, Halfacree, 2001, Hoggart, 1997, Phillips, 1993 ), no ocupan un lugar de reflexión dentro de la geografía española. Como tampoco la incidencia de estos cambios en la estructura social sobre la política municipal y el gobierno de amplios espacios territoriales (Cloke, Little, 1990; Cloke, Phillips, Thrift, 1995).

La despoblación del campo como eje de estudio sobre el poblamiento de España ha sido seguido en la actualidad por una literatura abundante aunque con un desigual reparto geográfico sobre los procesos de periurbanización o extensión de la ciudad y sus habitantes en áreas próximas a la siempre confusa frontera que divide y liga urbano y rural (Camarero, 1993; Estébanez, 1991; García Ballesteros, Pozo, 1991; Monclús (ed), 1998; Santos Preciado, 2001). Sin embargo, las transformaciones que experimenta el poblamiento y que en determinadas grandes áreas metropolitanas van ligadas a un proceso creciente de difusión de la población por migración parece que hacen conveniente plantear una nueva escala que tome en consideración los cambios, impactos e incidencias que la extensión de la ciudad y sus habitantes puede tener sobre áreas territoriales más amplias y, en este sentido, afectar de lleno a las que se definen como áreas rurales.

En esta comunicación se presenta una primera aproximación al caso de Cataluña. El análisis se centra en los datos disponibles sobre población y migraciones que desde la década de los noventa es posible obtener a escala municipal para el conjunto de municipios catalanes. Y toma como ámbitos prioritarios de estudio las comarcas y la clasificación de los municipios según tamaño. La existencia de un sistema urbano dinámico y consolidado sobre el que domina el área metropolitana de Barcelona (figura 1) proporciona un marco de interés para la observación de dicho proceso de difusión de la población y, seguramente a partir de estudios más detallados y con mayor aportación por parte del trabajo de campo sobre el propio territorio, se podrán delimitar las nuevas pautas de localización de la actividad económica, la población y la configuración social sobre el territorio y, en particular, sobre el espa- 
cio rural catalán. La nueva configuración de lo que parece ser una nueva forma de poblamiento, de interrelación con el territorio y dè cambio social y económico lleva a plantear ciertos interrogantes y líneas de investigación sobre la transformación territorial y, en especial, la incidencia que dichos cambios tiene sobre la configuración social en los pequeños municipios, el campo, su vinculación con la ciudad y las áreas urbanas y su posición en la jerarquización del territorio.

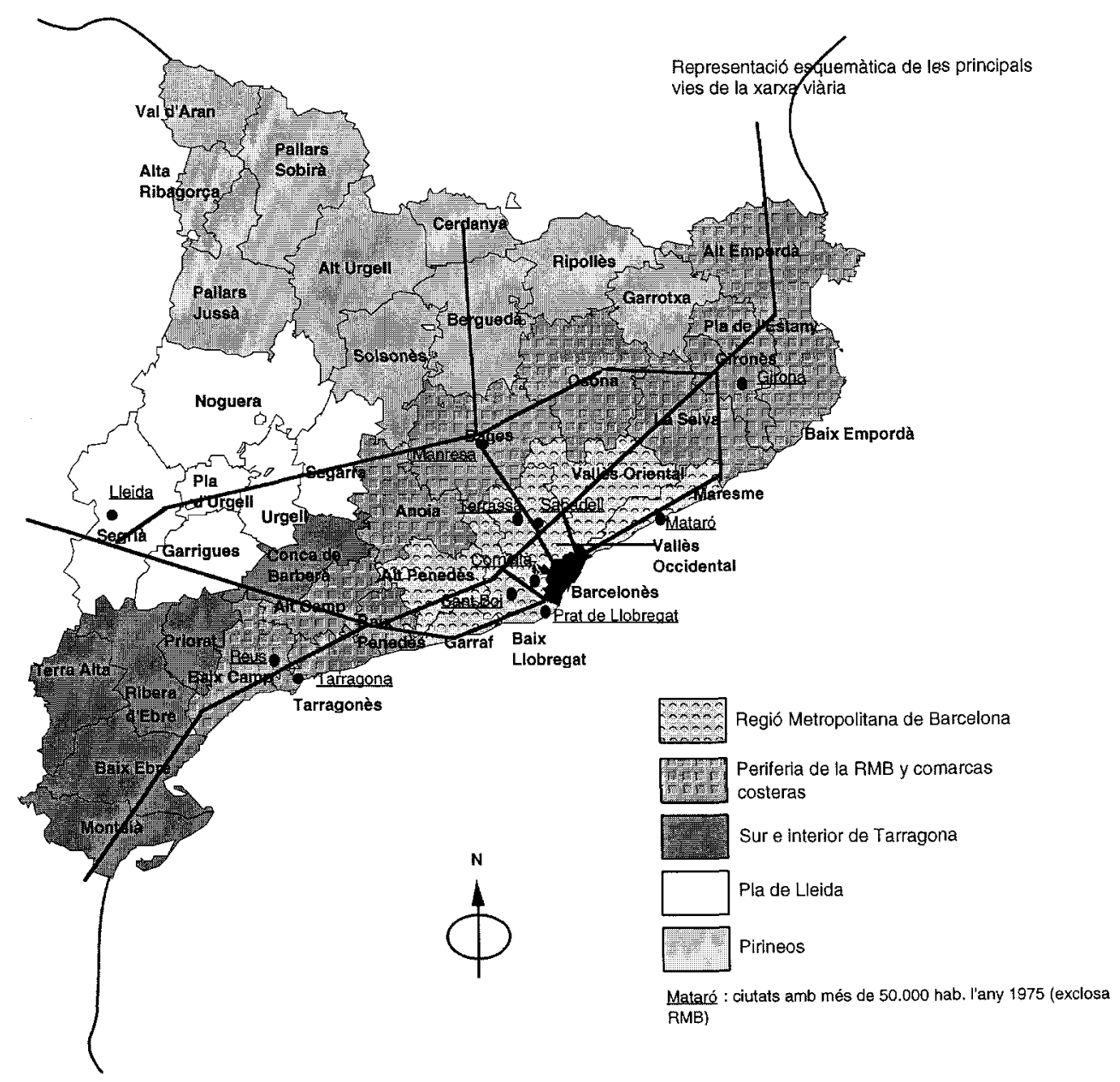

Figura 1. Comarcas y áreas de Cataluña. 


\section{De la concentración a la dispersión de población: la cascada contra- urbanizadora y sus límites territoriales}

El crecimiento continuado durante todo el siglo XX que experimentó la población catalana alcanzó momentos de crecimiento muy intensos que, sin embargo, se han ido moderando desde los años setenta hasta llegar a una cierta tendencia a la estabilización de la población desde la segunda mitad de la década de los años setenta, cuando Cataluña se acercó a la barrera de los seis millones de habitantes según los datos del censo de 1981 (tabla 1). Tendencia que parece que se vuelve a romper durante el último quinquenio, 1996-2001, con un crecimiento significativo producido básicamente por la inmigración extranjera.

Una de las áreas más estudiadas, debido a su población y su importancia económica, la constituye Barcelona y los municipios próximos (Durà, 1995; Mendizàbal, 1992; Módenes, 1992 y 1995; Nel.lo, 1995 y 1998a, 1998b; Pujadas et al., 1991). Como indica Nel.lo (1998a), un hecho significativo en la década de los ochenta es que se rompió la continuidad en el proceso de concentración de población en la Región Metropolitana de Barcelona -en adelante, RMB-, aunque a la luz de los últimos datos y tal como sucede con el conjunto de Cataluña dicho proceso parece que retoma otra vez un nuevo impulso. Sin embargo, esta ralentización del crecimiento se produjo paralelamente al proceso de redistribución interna de la población dentro del área metropolitana y en menor medida en el conjunto de Cataluña, actuando el Barcelonès como impulsor de estos cambios. Las pérdidas de población entre 1991 y 1996 fueron especialmente abundantes, del orden de las 27.000 personas anualmente.

Una parte cada vez más grande de la población se desplaza a vivir a municipios más periféricos en un proceso de creciente difusión de la ciudad sobre el territorio, en lo que se podría conceptualizar como un proceso de cascada contraurbanizadora (Champion, Coombes, Fotheringham, 1998). Barcelona y los municipios más próximos que la rodean pierden población frente a la primera y, cada vez más, segunda corona metropolitana (Ajenjo et al., 1993; Módenes, Pascual, 1998). La RMB consigue mantener el volumen de población y de alguna manera las pérdidas del Barcelonès quedan casi totalmente compensadas por las ganancias del resto de la RMB. Aunque también los municipios más grandes de esta segunda corona (Sabadell, Terrassa, Mataró y Granollers) no crecen o incluso pierden población. Una transformación del poblamiento debido, en buena medida, a la creciente movilidad intrametropolitana, donde los cambios residenciales cada vez se hacen a una distancia más elevada del lugar de origen, la direccionalidad predominante son las áreas menos densas, de poblamiento más difuso y donde el factor explicativo principal lo constituye el mercado de la vivienda mientras que queda en un plano secundario el laboral (Nel.lo, 1998a). 


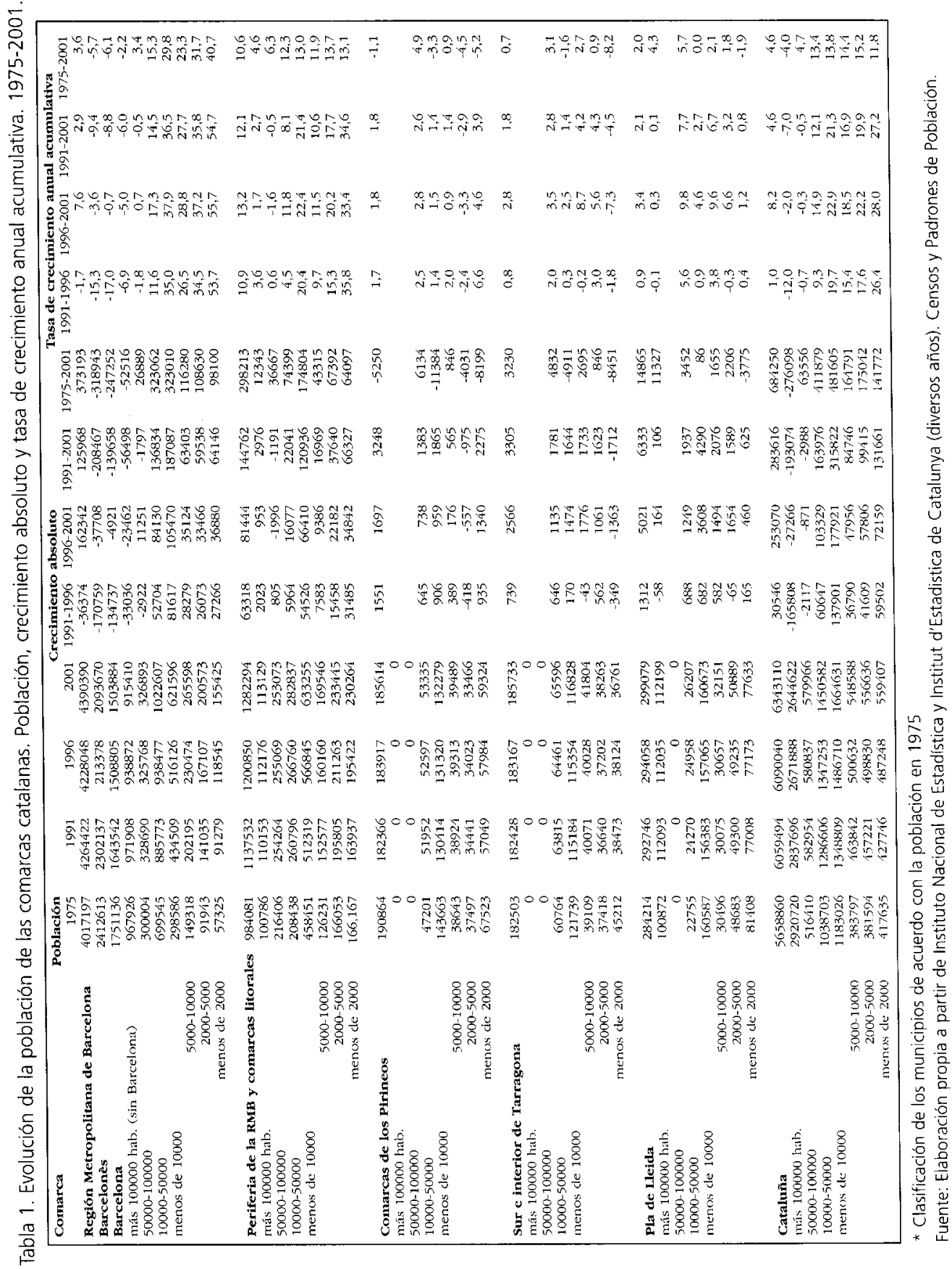


La desagregación de los datos de población según el tamaño de municipio introduce unos matices interesantes sobre la evolución del proceso de dispersión de la población y su impacto territorial en el ámbito metropolitano. Barcelona y los municipios con más de 50.000 habitantes de la RMB muestran una tendencia clara hacia la estabilización o el decrecimiento de la población. Las dos únicas categorías con un crecimiento absoluto de la población-del orden de los 300.000 habitantes entre 1975 y 2001- y también relativo son las que se podría cualificar como pequeñas y medianas ciudades y, dentro de las prevenciones que cabría poner a este término, los municipios rurales de la RMB. En este sentido, merece la pena destacar la evolución experimentada por los municipios menores de 2.000 habitantes, que casi llegan a triplicar su población.

Pero, fuera de este ámbito metropolitano, ¿qué sucede en el resto de Cataluña?. Una primera constatación interesante viene de la suavización de la polaridad o dualidad que se daba en el territorio catalán antes de la década de los ochenta entre comarcas con crecimiento y comarcas con decrecimiento, comarcas con saldo migratorio positivo y comarcas con saldo migratorio negativo. Actualmente hay una mayor heterogeneidad de las evoluciones (Ajenjo et al., 1993; Módenes, Pascual, 1998). De todas maneras, esta superación de evoluciones tan contrapuestas no puede hacer perder de vista que todavía hay una gran parte del territorio que padece una pérdida constante de población motivada ya sea por un crecimiento natural negativo o un crecimiento natural positivo pero cada vez más raquítico, ya sea por la continuidad del saldo migratorio negativo (Aldomà, 1999).

Fuera del ámbito estrictamente metropolitano las únicas ciudades que superaban en el año 1975 la barrera de los 50.000 habitantes, y que de hecho son las mismas que en la actualidad, se limitan a cinco. Las capitales de provincia: Lleida, Tarragona y el conjunto Girona más Salt, que pese a la desagregación posterior se ha mantenido agregado para poder seguir la serie desde 1975, más Manresa y Reus. Los dos casos de crecimiento claro de la población se encuentran, por una parte, en las comarcas periféricas a la RMB y, en segundo lugar y de manera más débil y con dudas respecto a su continuidad en el futuro, en los Pirineos.

Los municipios que rodean la RMB y los que se sitúan a lo largo de la costa central y norte, la más vinculada con las ciudades de Barcelona-Girona-Tarragona, mantienen un ritmo de crecimiento positivo aunque normalmente por debajo de las comarcas de la RMB, como se ha comentado anteriormente. También es posible observar la correlación negativa, en términos generales, entre tamaño del municipio y evolución de la población, aunque en este caso y a diferencia de los municipios de la RMB todos los rangos de municipio crecen en mayor o menor medida. En este caso, los mayores crecimientos se registran en los municipios de menos de 50.000 habitantes. 
Sin embargo, fuera de la RMB y las comarcas próximas no es posible observar las mismas tendencias respecto a la difusión de la población. Si se toma como referencia el último cuarto de siglo ha continuado el proceso de concentración en los municipios más grandes: en este caso los que se sitúan entre 10.000 y 50.000 habitantes debido a que no los hay con más población, excepto el caso puntual de Lleida. Por tanto, un mayor nivel de desglose geográfico permite apreciar las dinámicas diferentes que se dan en el territorio y que quedarían ocultas si sólo se atiende el caso de los tamaños municipales para el conjunto de Cataluña.

Por lo que respecta al área de los Pirineos y debido a su singularidad y a la posible aparición de nuevas tendencias por lo que respecta a la evolución de la población, es conveniente destacar que la pérdida de población se ralentiza durante la década de los noventa e incluso es posible detectar una evolución positiva en los municipios más pequeños, los de menos de 2.000 habitantes, entre 1996-2001. Seguramente, un análisis más pormenorizado territorialmente dibujaría las diferencias entre municipios con relación a su especialización económica y al impacto que la promoción de nuevas formas de turismo tiene en determinados municipios y parajes, que se ven afectados más directamente, caso por ejemplo de las comarcas de la Val d'Aran y la Cerdanya. Resta por saber si estos cambios son meramente coyunturales o apuntan hacia un crecimiento sostenido y continuado en el futuro de unas áreas muy castigadas históricamente por los procesos de despoblación.

\section{La redefinición del sistema migratorio catalán: la migración interior como motor de la dispersión de la población}

Una primera constatación es el incremento generalizado de las tasas de migración para todas las comarcas y, en especial, durante el último quinquenio ${ }^{2}$ (tabla 2). Ahora bien, se ha de tener en cuenta que se partía de unos niveles bajos a principios de los años ochenta, como consecuencia del impacto de la crisis económica. Y por otra parte, se ha de matizar el incremento desde 1986, debido a la mejora en el sistema de registro de las altas y bajas municipales.

Las tasas de inmigración más elevadas se extienden en el eje formado por las comarcas litorales de Gerona, las comarcas de la RMB -exceptuando el Barcelonèspara extenderse hasta el Baix Camp, con un máximo que se mantiene de forma constante y que corresponde al Baix Penedès. Otros máximos se registran en ciertas comarcas de los Pirineos. La Cataluña que se podría definir como menos inmigratoria corresponde a las comarcas interiores. La dinámica de la emigración parece más errática, aunque cada vez tiende a acercarse a las tasas de inmigración. Esta corres- 
Tabla 2. Inmigración, emigración y saldo de los municipios de Cataluña según tamaño. 1992-2000.

\begin{tabular}{|c|c|c|c|c|c|c|}
\hline & Inmigración & Emigración & $\begin{array}{c}\text { Saldo } \\
\text { migratorio }\end{array}$ & Inmigración & migración & $\begin{array}{l}\text { Saldo } \\
\text { migratorio }\end{array}$ \\
\hline Región Metropolitana de Barcelona & 882590 & 950637 & -68047 & 26,1 & 28,1 & $-2,0$ \\
\hline Barcelonès & 240468 & 469809 & -229341 & 14,1 & 27,6 & $-13,5$ \\
\hline Barcelona & 136596 & 313132 & -176536 & 11,3 & 25,9 & $-14,6$ \\
\hline más de 100000 hab. (sin Barcelona) & 129756 & 196981 & -67225 & 17,3 & 26,2 & $-9,0$ \\
\hline $50000-100000$ & 51951 & 64442 & -12491 & 19,9 & 24,7 & $-4,8$ \\
\hline $10000-50000$ & 285435 & 231935 & 53500 & 38,0 & 30,9 & 7,1 \\
\hline $5000-10000$ & 94584 & 58929 & 35655 & 51,3 & 32,0 & 19,3 \\
\hline menos de 5000 & 172696 & 85218 & 87478 & 75,6 & 37,3 & 38,3 \\
\hline Periferia RMB y comarcas litorales & 333016 & 261974 & 71042 & 34,7 & 27,3 & 7,4 \\
\hline más de 100000 hab. & 21449 & 16153 & 5296 & 23,9 & 18,0 & 5,9 \\
\hline $50000-100000$ & 40928 & 38901 & 2027 & 20,1 & 19,1 & 1,0 \\
\hline $10000-50000$ & 61222 & 55382 & 5840 & 28,7 & 26,0 & 2,7 \\
\hline $5000-10000$ & 42024 & 35732 & 6292 & 32,8 & 27,9 & 4,9 \\
\hline menos de 5000 & 167393 & 115806 & 51587 & 51,5 & 35,6 & 15,9 \\
\hline Comarcas del Pirineo & 39725 & 34651 & 5074 & 27,0 & 23,6 & 3,4 \\
\hline más de 100000 hab. & 0 & 0 & 0 & & & \\
\hline $50000-100000$ & 0 & 0 & 0 & & & \\
\hline $10000-50000$ & 8204 & 7659 & 545 & 19,5 & 18,2 & 1,3 \\
\hline $5000-10000$ & 7857 & 8036 & -179 & 25,0 & 25,6 & $-0,6$ \\
\hline menos de 5000 & 22829 & 18271 & 4558 & 31,0 & 24,8 & 6,2 \\
\hline Sur e interior de Tarragona & 30272 & 25686 & 4586 & 20,7 & 17,5 & 3,1 \\
\hline más de 100000 hab. & 0 & 0 & 0 & & & \\
\hline $50000-100000$ & 0 & 0 & 0 & & & \\
\hline $10000-50000$ & 6715 & 6299 & 416 & 13,0 & 12,2 & 0,8 \\
\hline $5000-10000$ & 7132 & 5757 & 1375 & 22,3 & 18,0 & 4,3 \\
\hline menos de 5000 & 16425 & 13630 & 2795 & 27,3 & 22,6 & 4,6 \\
\hline Pla de Lleida & 48171 & 46199 & 1972 & 20,5 & 19,6 & 0,8 \\
\hline más de 100000 hab. & 16095 & 13693 & 2402 & 18,0 & 15,3 & 2,7 \\
\hline $50000-100000$ & 0 & 0 & 0 & & & \\
\hline $10000-50000$ & 4419 & 3964 & 455 & 22,1 & 19,9 & 2,3 \\
\hline $5000-10000$ & 5612 & 5474 & 138 & 22,9 & 22,3 & 0,6 \\
\hline menos de 5000 & 22045 & 23068 & -1023 & 21,8 & 22,8 & $-1,0$ \\
\hline Cataluña & 1333774 & 1319147 & 14627 & 27,4 & 27,1 & 0,3 \\
\hline más de 100000 hab. & 303896 & 539959 & -236063 & 14,2 & 25,3 & $-11,0$ \\
\hline $50000-100000$ & 92879 & 103343 & -10464 & 20,0 & 22,2 & $-2,3$ \\
\hline $10000-50000$ & 365995 & 305239 & 60756 & 34,0 & 28,3 & 5,6 \\
\hline $5000-10000$ & 157209 & 113928 & 43281 & 39,3 & 28,4 & 10,8 \\
\hline menos de 5000 & 401388 & 255993 & 145395 & 50,9 & 32,5 & 18,4 \\
\hline
\end{tabular}

Fuente: Elaboración propia a partir de Institut d'Estadistica de Cataluña (diversos años), Estadística de Variaciones Residenciales y Censos y Padrones de Población.

pondencia se da en las comarcas pirenaicas y, cada vez de forma más clara, en las comarcas de la RMB y las que le rodean. Todas ellas forman el conjunto de áreas con niveles de emigración más elevados. 
La migración interna catalana está en gran parte determinada por las pulsaciones migratorias del Barcelonès, la cual fluctúa alrededor de un tercio del total de las bajas registrada en Cataluña y cerca del $50 \%$ sobre el total de los flujos migratorios que traspasan los límites comarcales. El Barcelonès se sitúa entre las comarcas con una mayor tasa de emigración si no se tiene en cuenta la migración dentro de la propia comarca $(15,0 \% 0)$, pero no establece la tasa máxima ya que es superada por la comarca con mayor capacidad de atracción de población de toda Cataluña, el Baix Penedès (19,2\%0), y se sitúa en el mismo nivel que algunas comarcas de los Pirineos. Las comarcas con unas tasas de inmigración más elevadas son las próximas al Barcelonès: Alt Penedès, Baix Llobregat, Baix Penedès, Garraf y Tarragonès en la costa sur; Maresme, La Selva, el Vallès Oriental y el Baix Empordà, en la costa norte. Todas ellas con un consolidado desarrollo urbanístico, creación de segundas residencias y urbanizaciones. La importancia del Barcelonès como comarca que condiciona de manera significativa la migración interna de Cataluña queda de relieve si tenemos en cuenta que sólo tres comarcas mantenían un saldo migratorio negativo con el resto de Cataluña: Barcelonès, Noguera y Segrià. Y sólo en el caso del Barcelonès el dato es destacable $(-10,7 \%$ ).

El estudio de la migración no por comarcas sino según el tamaño de los municipios muestra elementos de interés que apuntan y confirman la relación existente entre la migración, el saldo migratorio y, por tanto, la evolución de la población. En este sentido, la migración muestra una mayor capacidad para apuntar el nivel de atracción de los diferentes municipios, la cual quedaba más oculta a veces en el caso de la consideración de la evolución de la población. El sesgo que introduce la estructura por edades de la población y en especial el envejecimiento de aquellos municipios del interior de Cataluña, en áreas de predominio rural y agrario, ha producido unas estructuras por edad fuertemente envejecidas, que dan como resultado un decrecimiento de la población aún teniendo una entrada neta positiva derivada de la migración. Quizás, el ejemplo más claro se da en las comarcas y municipios de los Pirineos. Es interesante destacar el hecho de que el saldo migratorio de los municipios de menos de 5.000 habitantes durante la década de los noventa es consistentemente positivo. Y superior al que registran los municipios del Pla de Lleida y del sur e interior de Tarragona.

Las dinámicas de mayor crecimiento inmigratorio se dan en los municipios de menor tamaño tanto de la RMB (de 5.000 a 10.000 hab. y menos de 5.000 hab.) como de la periferia de la RMB (menos de 5.000 hab.). Las tasas de inmigración doblan las que registra el conjunto de Cataluña o la RMB considerada en su totalidad. Y ello junto a unas tasas de emigración relativamente elevadas pero próximas a la media de Cataluña produce que sean los únicos grupos de municipios con un saldo migratorio claramente positivo. Seguramente los problemas de subestimación y falsos registros que afecta a la estadística de variaciones residenciales introduce elementos que invitan a tomar estos resultados con prudencia. Sin embargo, fuera de los matices y la precisión exacta es interesante constatar como a grandes rasgos se produce este crecimiento de la población y, en este caso, como consecuencia de la inmigración. 


\section{Recapitulación y reflexiones en torno a la transformación del sistema de poblamiento en Cataluña}

El proceso de difusión de la población aparece claramente consolidado en el caso de Cataluña. Si bien la RMB fue en un primer momento el área de mayor crecimiento de población, en la actualidad la periferia de esta región metropolitana se consolida como una de las principales áreas de recepción. En general, la totalidad de la línea costera y determinados valles de los Pirineos se suman a esta capacidad de atracción de la población. El turismo, la construcción y en general las actividades ligadas a la provisión de servicios a la población actúan dinamizando la economía de estas áreas. Pero tampoco se ha de descuidar la extensión del proceso de ocupación residencial de toda la franja costera que en la actualidad se extiende de forma cada vez más incisiva hacia el interior, hacia espacios menos afectados hasta ahora por el desarrollo turístico y la construcción masiva de residencias. Especialmente importante por las posibles tendencias de cambio que se apuntan es la dinámica de algunos municipios de los Pirineos. La recuperación demográfica acompaña la implantación y desarrollo de una actividad turística creciente y diversificada que permite un asentamiento y atracción de población.

Las tendencias generales en la evolución del poblamiento tienden a primar los municipios más pequeños. En este sentido, la relación entre evolución de la población o nivel de inmigración muestra la correlación existente con el tamaño de los municipios. Son aquellos por debajo de los 10.000 habitantes, los que a efectos estadísticos se conceptualizan como municipios rurales o intermedios, los que presentan un mayor crecimiento de la población y unas mayores tasas de inmigración y saldo migratorio positivo.

Uno de los elementos que desde la literatura internacional se toman como principal referencia para la explicación de estas pautas de migración y poblamiento es la vivienda. Las preferencias residenciales, la calidad de vida ambiental pero también social, que se supone en el campo, y la dinámica de precios de la vivienda son elementos de primer orden para explicar estas dinámicas. En el caso español y catalán falta una mayor atención a estos factores no sólo por lo que respecta a la vinculación entre el mercado de la vivienda, la migración y la distribución espacial de los grupos sociales, sino también por lo que respecta a las casuísticas que se esconden detrás de esta migración al "campo", el sistema de preferencias y constricciones que da lugar a un poblamiento difuso. En este sentido, el estudio de la promoción residencial de nuevas viviendas y la relación de estas promociones y los crecimientos que experimentan los municipios con el sistema de infraestructuras de comunicación son temas de interés para un estudio más pormenorizado no sólo en el caso de Cataluña sino en el conjunto de España. Y desde un punto de vista cultural también seria interesante analizar como se forman, modelan y dirigen las preferencias residenciales de la 
población. El idilio de lo rural presente en otros países puede ser un elemento detrás de esta migración hacia las áreas rurales y los municipios más pequeños del país. Un proceso con una fuerte repercusión en la transformación actual y seguramente futura del territorio. La delimitación espacial y los volúmenes de población que se encuentran implicados en estos cambios ya suponen una importante parcela de estudio.

Más dificultades reviste, pero también puede ofrecer unos resultados interesantes el estudio de la composición y transformación social del medio rural. Los estudios efectuados en otros países han tendido a acentuar la inmigración de los grupos sociales más cualificados, los procesos de gentrificación social y el desplazamiento de los habitantes que vivían con anterioridad estos espacios. Esta dualización entre recién llegados y autóctonos supone, sin embargo, una simplificación de una situación más compleja en la que los intereses y las divergencias cruzan y permeabilizan los diferentes sectores sociales. Asimismo la recomposición social del campo muestra una mayor complejidad en los desplazamientos de la población.

\section{Notas}

1 Esta comunicación es el resultado de la investigación llevada a cabo bajo el marco de la beca postdoctoral Batista i Roca de la Generalitat de Cataluña. La base de datos fue creada en el Sussex Centre for Migration Research de la University of Sussex.

2 Los datos disponibles de flujos comarcales tan sólo alcanzan un perído de veinte años. Los primeros datos corresponden al 1982 y los últimos al 2000. No se dispone de información de los años censales o padronales que se consideran defectuosos. Los datos para los años noventa sólo tienen en cuenta los movimientos internos -entre el resto de España y Cataluña o internos en Cataluña-. Se ofrecen de manera desagregada los datos de la inmigración proveniente del extranjero y no hay ninguna información sobre la emigración hacia el extranjero. Las tasas y saldos migratorios que se han calculado y que se presentan a continuación se han elaborado a partir de esta estadística de variaciones residenciales. Sería interesante, como ejercicio metodológico y para contrastar esta fuente, comparar estos resultados con los saldos migratorios resultantes de la ecuación compensatoria.

\section{Bibliografía}

AGE (sin fecha), From traditional countryside to postproductivism: recent trends in rural Geography research in Britain and Spain (1er Simposium de Geógrafos Rurales Británicos y Españoles), AGE.
Ajenjo, M., Arribas, R., Blanes, A., Mendizàbal, E., Módenes, J.A. (1993): La població de Catalunya, 1986-1991, Papers de Demografia, 74, (monográfico). 
Aldomè, I. (1999): La crisi de la Catalunya rural. Una geografia dels desequilibris comarcals (1960-1991), Lleida, Pagès.

Camarero, L. A. (1993): Del éxodo nural y del éxodo urbano. (Ocaso y renacimiento de los asentamientos rurales en España), Madrid, Ministerio de Agricultura, Pesca y Alimentación.

Champion, T., Coombes, M., Fotheringham, S. (dirs.) (1998): Urban exodus, Londres, CPRE.

Cloke, P. (1977): An index of rurality for England and Wales, Regional Studies, ?? p. $31-46$.

Cloke, P., Thrift, N. (1990): Class and change in rural Britain, en Rural restructuring (global processes and their responses), $\mathrm{T}$. Mardsen, P. Lowe, S. Whatmore (eds.), Londres, David Fulton, p. 165-181.

Cloke, P., Philips, M., Thrift, N. (1995): The new middle classes and the social constructs of rural living, en Social change and the middle class, T. Butler, M. Savage (eds.), Londres, UCL Press, p. 220-238.

Cloke, P., Little, J. (1990): The mural state? Limits to planning in rural society, Oxford, Clarendon Press.

Durà, A. (1995): Mobilitat residencial, contraurbanització $i$ canvi en l'estructura social de Santa Coloma de Gramanet (un municipi de la primera periferia metropolitana de Barcelona), Tesis doctoral, Departament de Geografia, Universitat Autònoma de Barcelona, Bellaterra (Barcelona). (document no publicat).

Estébanez, J. (1981): El proceso de urbanización del medio rural madrileño, en Estudios de Geografia. Homenaje a Alfredo
Aristán, Pamplona, Diputación Foral de Navarra, p. $151-168$.

Estébanez, J. (1988): Los espacios rurales, Geografia ITumana, R. E. Puyol, J., Mendoza, R., Madrid, Cátedra, p. 239-261.

Fielding, A.J. (1990): Counterurbanisation: threat or blessing?, en Western Europe: Challenge and change, D. Pinder (ed.), Londres, Belhaven, p. 226-239.

García Ballesteros, A., Pozo, E. (1991): Changements socio-démographiques dans la frange périurbaine de Madrid, Espace, Populations, Sociétés, 2, p. 309-323.

García Ramón, D., Tulla, T., Valdovinos, N. (1995): Geografia rural, Madrid, Síntesis.

Halfacree, K.H. (1991): The importance of spatial representations in residential migration to mral England in the $1980 \mathrm{~s}$ (A quest for "sopbisticated simplicity" in a postmodern world?), Tesis doctoral, Department of Geography, Lancaster University, Lancaster.

Halfacree, K.H. (1993): Locality and social representation: space, discourse and alternative definitions of the rural, Journal of Rural Studies, 1, p. 23-37.

Halfacree, K.H. (1994): The importance of 'the rural' in the constitution of counterurbanization: Evidence from England in the 1980s, Sociologia Ruralis, 2-3, p. 164-189.

Halfacree, K. (1995): Talking about rurality: social representation of the rural as expressed by residents of six English parishes, Journal of Rural Studies, 11, p $1-20$.

Halfacree, K. (1997): Contrasting roles for the post-productivist countryside. A postmo- 
dern perspective on counterurbanisation, en Contested countryside cultures. Otberness, marginalisation and nurality, P. Cloke, J. Little (eds.), Londres, Routledge, p. 70-93

Halfacree, K. (2001): Going 'back-to-land' again: extending the scope of counterurbanisation, Espace, Populations, Societes, 1-2, p. $161-170$

Hoggart, K. (1988): Not a definition of rural, Area, 20, p. 35-40.

Hoggart, K. (1990): Let's do away with rural, Journal of Rural Studies, 6, p. 245-257.

Hoggart, K. (1997): The middle classes in rural England 1971-1991, Journal of Rural Studies, 3, p. 253-273.

INRA-INSEE (1998): Les campagnes et leurs villes, París, INSEE-INRA.

Kayser, B. (1990): La renaissance rurale. (Sociologie des campagnes du monde occidental), Paris, Armand Colin.

Kayser, B. (dir.) (1993): Naissance de nouvelles campagnes, Marsella, Datar/Éditions de l'Aube.

Mendizábal, E. (1992): Els moviments migratoris a la Regió Metropolitana de Barcelona, en Enquesta de la Regió Metropolitana de Barcelona 1990, M. Subirats (dir.), Barcelona, Instituto de Estudios Metropolitanos de Barcelona.

Módenes, J.A. (1992): Nuevas herramientas para el análisis de la distribución de la población y el poblamiento en el territorio: la Región Metropolitana de Barcelona 19811991, Papers de Demografia, 67, p. 1-17.

Módenes, J.A. (1995): Anàlisi geodemogràfica de les àrees de relació migratòria local a la Regió Metropolitana de Barcelona, Memoria de Investigación Departament de Geografia, Universitat Autònoma de Barcelona, Bellaterra (Barcelona). (documento no publicado)

Módenes, J.A., Pascual, J. (1998): Les migracions interiors a Catalunya. Intensitat, selectivitat i estructura espacial dels fluxos migratoris, en La societat catalana, S. Giner (dir.), Barcelona, Institut de Estadística de Cataluña, p. 201-219.

Monclús, F. J. (ed.) (1998): La ciudad dispersa. Suburbanización y nuevas periferias, Barcelona, CCCB.

Murdoch, J. (1995): Middle-class territory? Some remarks on the use of class analysis in rural studies, Environment and Planning $A$, vol. 27 , p. $1213-1230$.

Nel.lo, O. (1995): Dinàmiques territorials i mobilitat urbana a la regió metropolitana de Barcelona, Papers. Regió Metropolitana de Barcelona, 24, p. 9-37.

Nel.lo, O. (1998a): Les dinàmiques metropolitanes: la difusió de la ciutat sobre el territori, en La societat catalana, S. Giner (dir.), Barcelona, Institut de Estadística de Cataluña, p. 307-329.

Nel.lo, O. (1998b): Los confines de la ciudad sin confines. Estructura urbana y límites administrativos en la ciudad difusa, en $L a$ ciudad dispersa, F.J. Monclús (ed.), Barcelona, Centre de Cultura Contemporània de Barcelona, p. 35-57.

Philips, M. (1993): Rural gentrification and the processes of class colonisation, Journal of Rural Studies, 2, p. 123-140.

Pujadas, I., Arribas, R., Mendizàbal, E., Módenes, J.A., Miret, P. (1991): Moviments 
migratoris intermunicipals a la Regió Metropolitana de Barcelona, Papers de Demografia, 56, (monográfico).

Santos Preciado, J. M. (2001): El proceso de distribución espacial de la población en las periferias metropolitanas españolas (1960-
1996), Anales de Geografia de la Universidad Complutense de Madrid, 21, p. 139-182.

Urry, J. (1995): A middle-class countryside?, en Social change and the middle class, T. Butler, M. Savage (eds.), Londres, UCL Press, p. 205-219. 